\title{
Une recherche exploratoire pour accéder à la motivation de l'apprenant universitaire en formation à l'enseignement dans son expérience de formation en ligne
}

Caroline Bourque et Céline Garant

\section{(2) OpenEdition Journals}

\section{Édition électronique}

URL : http://journals.openedition.org/trema/3400

DOI : 10.4000/trema.3400

ISSN : 2107-0997

\section{Éditeur}

Faculté d'Éducation de l'université de Montpellier

\section{Édition imprimée}

Date de publication : 1 mai 2016

Pagination : 65-78

ISBN : 979-10-96627-00-4

ISSN : $1167-315 X$

\section{Référence électronique}

Caroline Bourque et Céline Garant, « Une recherche exploratoire pour accéder à la motivation de l'apprenant universitaire en formation à l'enseignement dans son expérience de formation en ligne ", Tréma [En ligne], 44 | 2016, mis en ligne le 01 juin 2016, consulté le 21 décembre 2020. URL : http:// journals.openedition.org/trema/3400; DOI : https://doi.org/10.4000/trema.3400 


\title{
Une recherche exploratoire pour accéder à la motivation de l'apprenant universitaire en formation à l'enseignement dans son expérience de formation en ligne
}

\author{
Caroline Bourque et Céline Garant
}

\section{Une problématique à considérer}

Alors que la formation à distance (FAD) existe depuis la fin du XIXe siècle (Perriault, 2002), l'usage du numérique permet dorénavant d'élargir ses potentialités. La FAD est un terme générique se définissant comme un système de formation médiatisée où les étudiants n'ont pas à se déplacer sur un lieu de formation et peuvent se former sans temps prédéfini et sans la présence physique du formateur (Prata et Lopes, 2005). La formation en ligne, plus spécifiquement, fait partie des différentes modalités que peut prendre la FAD, mais elle nécessite, entre autres, l'utilisation du numérique et des médias interactifs (Kim, 2008). L'enseignement et l'apprentissage passent alors par un réseau Internet. Les formations en ligne, constituant ainsi la quatrième génération de FAD (Ibid.), augmentent rapidement partout dans le monde et gagnent en popularité (Kim, Barbier et Verrier, 2007). En fait, pour de plus en plus d'universités, ce mode de formation devient une alternative à l'enseignement en présentiel (Allen et Seaman, 2008; Bates, 2011). Les adultes constituent d'ailleurs $92 \%$ de la clientèle étudiante en ligne (Yoo et Huang, 2013). 


\section{I.1. Les taux d'abandon en formation en ligne}

2 Par contre, malgré cette augmentation de popularité, de nombreuses études recensent un fort taux d'abandon dans ce type de formation (Levy, 2007; Metzger et Delalonde, 2005) en comparaison avec les formations équivalentes en présentiel.

3 En effet, plusieurs études indiquent que le taux de persévérance des étudiants dans ce type de formation se situerait entre $30 \%$ et $50 \%$ (Kranzow, 2013, dans Lakhal et Khechine, 2014). Selon Levy (2007), « la littérature fait état d'un consensus clair sur le fait que l'abandon, surtout en formation à distance, est un phénomène difficile et embarrassant » (p. 187).

4 Le phénomène de l'abandon dans les formations en ligne au niveau universitaire est préoccupant. Les conséquences de ce manque de persévérance des étudiants sont multiples pour les étudiants eux-mêmes, les institutions et la société (Grayson, 2003; Sauve, Debeurme, Martel, Wright, Hanca, Fournier et Castonguay, 2007).

5 Il convient donc de réfléchir aux solutions possibles afin de favoriser la persévérance des étudiants universitaires en formation en ligne. Les causes d'abandon sont nombreuses et ont été étudiées par plusieurs chercheurs. Des modèles d'abandon et de persévérance en présentiel et en ligne ont d'ailleurs été élaborés (Kember ; 1989 ; Park, 2007 ; Rovai, 2003 ; Tinto, 1975). En effet, Tinto (1975) s'est d'abord intéressé à l'abandon des étudiants universitaires dans les formations en présentiel. Le modèle qu'il a développé a ensuite été repris par Kember (1989) qui l'a adapté pour le contexte spécifique de la FAD. Rovai (2003), pour sa part, a créé un modèle sur la persévérance des étudiants universitaires en formation en ligne. Enfin, Park (2007) a développé un modèle, sur la base de celui de Rovai (2003), pour le rendre spécifique à l'abandon des adultes en formation en ligne.

Concrètement, Park (2007) a créé son propre modèle spécifique à l'abandon des adultes dans les formations en ligne (figure 1), sur la base de celui de Rovai (2003). Dans ce dernier, on aperçoit les caractéristiques de l'apprenant (âge, sexe, éducation, statut d'emploi), ainsi que ses compétences. Ces deux aspects sont des éléments présents avant même que le cours commence et ils s'avèrent importants à considérer pour la persévérance. Des facteurs externes (situation familiale, problèmes financiers...) et internes (intégration sociale, manque de motivation...) s'avèrent également présents avant, mais aussi pendant le cours. Les différentes flèches démontrent les interrelations que Park (2007) a identifiées entre ces ensembles de facteurs. Enfin, cette dynamique mène l'étudiant à la persévérance ou à l'abandon. 
Figure 1 : Modèle théorique de l'abandon de l'adulte en formation en ligne (Park, 2007)

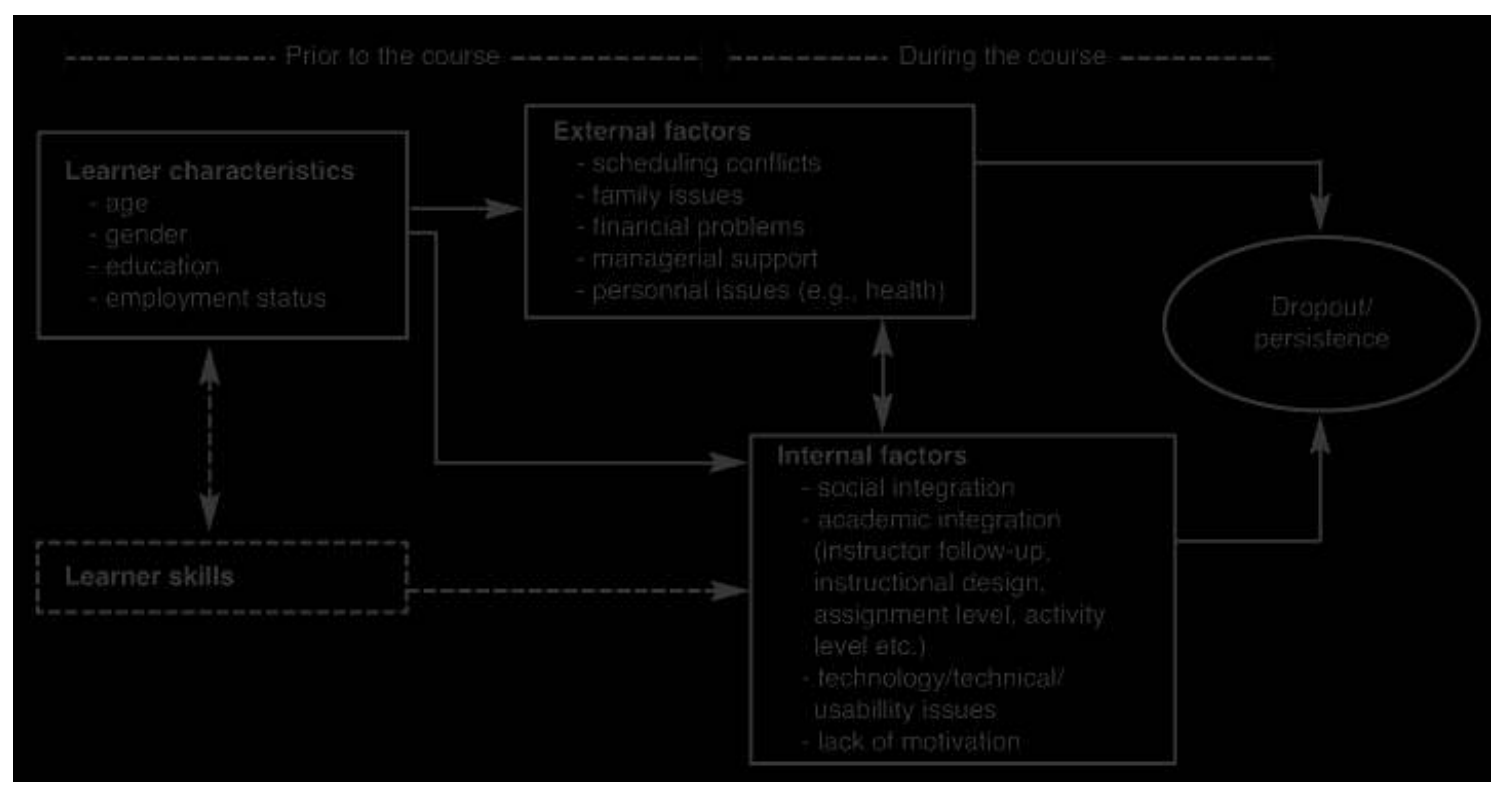

Ce modèle fait donc état des facteurs à risque et de protection pour la persévérance des adultes en formation en ligne. Également, on constate l'importance accordée aux facteurs internes. Le manque de motivation en fait partie.

Dans le même sens, certaines recherches ont été menées dans le but de favoriser la persévérance, spécifiquement dans le contexte universitaire des formations en ligne (Aragon et Johnson, 2008 ; Lee et Choi, 2011). Parmi ces différentes recherches, plusieurs abordent la question de la motivation (Castles, 2004 ; Ivankova et Stick, 2007 ; Levy, 2007 ; Park et Choi, 2009 ; Poellhuber et Chomiene, 2006 ; Racette, 2009), que ce soit sous l'angle de la cause (la démotivation comme facteur d'abandon) ou de l'intervention (stratégies pour favoriser la motivation afin d'inciter à la persévérance). Il est donc possible de constater qu'un lien les unit. Il nous semble donc important de nous intéresser à la nature de ce lien pour découvrir si la motivation doit être considérée lorsqu'il est question d'agir sur la persévérance.

\section{I.2. La motivation : une variable importante à prendre en compte}

La motivation est parfois évoquée dans les écrits comme le moteur de l'apprentissage (OCDE, 2004, dans Thouraya, 2007). Elle se révèle d'ailleurs être un facteur critique de cet apprentissage (Chen et Jang, 2010) et joue donc un rôle prépondérant au regard de la réussite (Poellhuber et Chomiene, 2006 ; Ruel, 2010). Racette (2009) parle de la motivation comme étant ce qui apporte l'élan nécessaire permettant la réalisation d'une tâche de façon satisfaisante. Elle ajoute que la motivation peut faire la différence entre subir et agir (Ibid.).

Sauve et al. (2007) rapportent que plusieurs études ont démontré un lien entre la motivation et la persévérance. Park et Choi (2009) ajoutent qu'il s'agit de la variable la plus souvent étudiée en lien avec l'abandon. Alors que la motivation est identifiée comme étant un facteur critique au regard de l'apprentissage (Lim, 2004), elle est d'autant plus importante dans le contexte de la formation en ligne. En effet, plusieurs études indiquent 
une corrélation significative entre la motivation de l'apprenant et la rétention dans les programmes en ligne (Castles, 2004 ; Ivankova et Stick, 2007).

11 Toutefois, même si de nombreuses recherches ont déjà été effectuées sur la motivation dans un contexte de formation en présentiel, Mullen et Tallent-Runnels (2006) ont découvert que les étudiants perçoivent les deux contextes (en ligne et en présentiel) différemment. Ainsi, « les chercheurs ne peuvent affirmer que les théories de motivation établies en présentiel traditionnel peuvent être directement transférées en ligne, sans justifications, parce que les caractéristiques de l'environnement d'apprentissage (flexibilité, accessibilité, communication médiée par ordinateur) et la dynamique motivationnelle des étudiants sont différents $»^{1}$ (Chen et Jang, 2010, p. 742). C'est donc ce qui justifie des recherches approfondies dans le domaine de la motivation des apprenants en contexte de formation en ligne (Ibid.).

Plusieurs études ont cherché à connaitre les facteurs contribuant à la motivation des étudiants universitaires en formation en ligne. Prenons l'exemple de Bekele (2010), qui a effectué une recension d'études entre 1995 à 2007 sur la motivation et la satisfaction des étudiants en formation en ligne. Il a relevé les services de soutien, les caractéristiques de la technologie, la qualité du cours, l'engagement et le format du cours comme étant des aspects qui soutiennent la motivation et la satisfaction des étudiants dans ce type de formation. Dans le même sens, Selvi (2010) a cherché à déterminer les facteurs pouvant contribuer à augmenter la motivation des étudiants dans les cours en ligne. L'enquête réalisée auprès de 60 doctorants a révélé que le processus d'enseignement-apprentissage, les compétences $\mathrm{du}$ formateur, l'attention $\mathrm{du}$ participant, l'environnement d'apprentissage en ligne et la gestion du temps affectent leur motivation. Ainsi, les étudiants sondés déclarent qu'un meilleur environnement d'apprentissage en ligne, un processus d'enseignement-apprentissage et des mesures d'évaluation améliorées contribueraient à augmenter leur motivation.

Les différentes études mettent en évidence le fait que de nombreux facteurs peuvent avoir un impact sur la motivation des étudiants en formation en ligne. Toutefois, Ruel (2010) est d'avis que les recherches qui mettent en relation l'abandon et des variables d'insatisfaction ne permettent pas de représenter le phénomène dans toute sa complexité puisqu'elles ne tiennent pas compte de l'expérience de l'étudiant. En effet, Bourdages (1994, dans Ibid.) propose d'analyser le poids des différentes variables internes et externes qui caractérisent l'expérience de l'étudiant, selon sa représentation et l'interprétation qu'il en fait. Ruel (Ibid.) ajoute que

L'élan individuel ne peut être compris de l'extérieur [...]. Ce qui incite un étudiant à persévérer dans ses études, ou à les abandonner, relève de l'étudiant, de ses affects, de son existence dans l'espace et le temps. De là, on peut comprendre que c'est sur l'expérience de celui-ci qu'il faille agir (p. 30-31).

\section{I.3. Vers une question préliminaire de recherche}

Cette idée de s'intéresser à l'expérience de l'étudiant dans un contexte de formation en ligne nous apparait pertinente pour mieux comprendre comment la motivation de l'étudiant se manifeste dans sa formation en ligne. En ce sens, il faut saisir le sens l'expérience qu'il vit et les facteurs qui influencent cette motivation, selon ce qui le caractérise, à ce moment précis de son parcours. 
16 Pour la recherche exploratoire, réalisée dans le cadre de notre thèse doctorale et présentée ici, nous proposons cette question préliminaire de recherche: Comment la motivation de l'apprenant universitaire se manifeste-t-elle dans son expérience de formation en ligne?

\section{La démarche méthodologique de la recherche exploratoire}

17 Afin de mieux cerner comment la motivation de l'apprenant universitaire se manifeste dans son expérience de formation en ligne, nous avons tout d'abord exploré les différentes dimensions sous-jacentes à un cours en ligne, afin de définir une base à partir de laquelle étudier l'expérience de l'apprenant dans un tel contexte de formation. C'est donc à partir des modèles de persévérance nommés plus tôt, de modèles connus de motivation $^{2}$, ainsi que de l'étude de 18 recherches empiriques ${ }^{3}$ portant sur la motivation des étudiants en formation en ligne, que nous avons élaboré un cadre synthèse qui nous servira de base pour notre recherche exploratoire.

18 En fait, ce cadre-synthèse, sans être un nouveau modèle de motivation, nous apparait intéressant comme grille d'analyse pour étudier l'expérience. Dans les prochaines sections, nous présentons d'abord ce cadre-synthèse, énumérant différents facteurs ayant un impact sur la motivation des apprenants en formation en ligne. Puis, nous précisons l'approche méthodologique et l'outil de collecte choisi, ainsi que la clientèle ciblée.

\section{II.1. Une proposition de cadre-synthèse pour une formation en ligne motivante}

19 Au regard des différentes études empiriques recensées et des modèles sélectionnés, nous avons développé un cadre-synthèse (figure 1) dans lequel, pour aborder la motivation, nous avons pris appui sur l'approche sociocognitive de Viau (2009). Dans une perspective interactionniste, Viau (Ibid.) considère la motivation de l'apprenant comme étant le résultat d'une interaction entre ses perceptions et des variables externes.

Ainsi, dans ce cadre-synthèse, quatre dimensions ont été mises en évidence pour représenter ces variables externes: le cours, le formateur, la technologie et les autres apprenants. Chacune de ces dimensions comprend d'ailleurs différents facteurs influençant la motivation de l'apprenant en formation en ligne. Dans ce cadre-synthèse, l'apprenant se trouve au centre.

Figure 1 : Cadre synthèse de motivation des apprenants universitaires en formation en ligne 


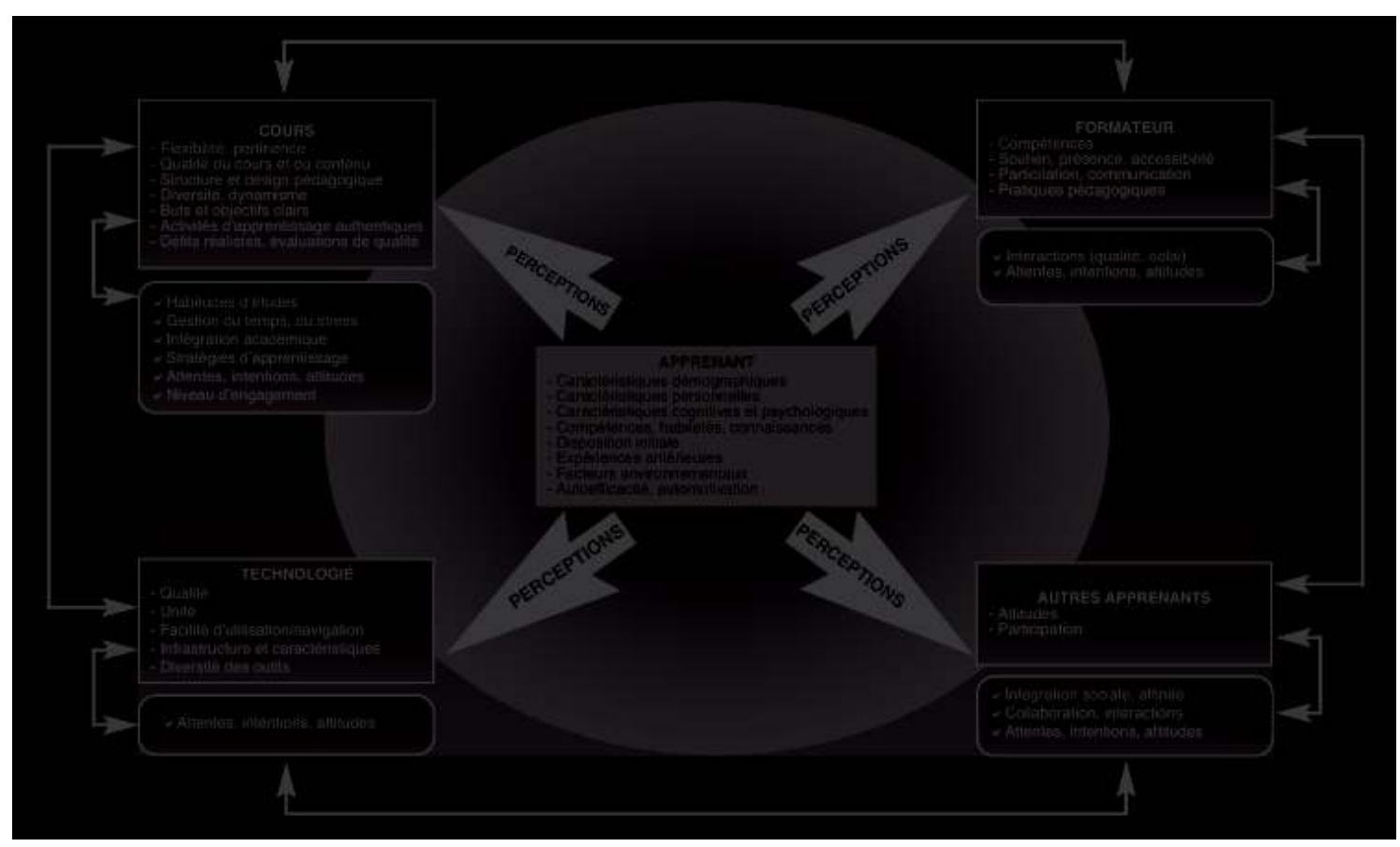

L'apprenant vit des interactions avec les variables externes (que nous appelons ici dimensions de la formation en ligne). Ce sont les perceptions que l'apprenant a des différents facteurs dans chacune de ces dimensions qui influencent sa motivation. Il est à noter que selon Viau (2009), «Les perceptions sont les jugements qu'une personne porte sur les événements, les autres et elle-même. Elles sont constituées à la fois d'un processus et de connaissances» (p. 22). Enfin, pour chacune de ces quatre dimensions, certains facteurs concernant les comportements de l'apprenant, comme ses habitudes d'étude, ses attitudes, ses interactions. Pour représenter cela, un encadré a été superposé à chacune des dimensions.

La théorie de Viau (Ibid.) s'appuie également sur le déterminisme réciproque de Bandura (1986, dans Ibid.) qui « propose d'étudier les phénomènes humains complexes, comme la motivation, en s'appuyant sur l'interaction qui existe entre les comportements d'une personne, ses caractéristiques individuelles et l'environnement dans lequel elle évolue » ( Ibid., p. 194).

23 Ainsi, notre cadre-synthèse s'appuie également sur ce principe. Les "caractéristiques individuelles» de la personne renvoient à la bulle centrale de l'apprenant; « l'environnement dans lequel elle évolue » est représenté par les quatre dimensions de la formation en ligne et; les "comportements d'une personne» réfèrent aux bulles superposées aux différentes dimensions. Ainsi, l'ensemble des composantes de ce cadre est en interaction et interinfluence (flèches bi-directionnelles) pour former un tout: la formation en ligne, représentée par le cadre en arrière-plan. Enfin, le grand cercle central devenant de plus en plus foncé tente d'illustrer la place grandissante de la motivation de l'apprenant. On peut supposer que plus ses perceptions deviennent positives, plus il s'approprie les différentes dimensions du cours et plus sa motivation tend à augmenter.

\section{II.1.1. L'apprenant}

Dès le début de sa formation en ligne, l'apprenant arrive avec ce qu'il est comme individu, son propre bagage: ses caractéristiques démographiques, personnelles, cognitives et 
psychologiques, ainsi que ses compétences, habiletés et connaissances. Ses dispositions initiales, ses expériences antérieures, que ce soit en ligne ou scolaires, ainsi que ses facteurs environnementaux, ont aussi une influence sur sa motivation. Finalement, plusieurs auteurs traitent de l'autoefficacité, soit la croyance que possède un individu en sa capacité de produire ou non une tâche, et de l'automotivation, soit la capacité de se motiver lui-même. Ainsi, la dimension centrale de l'apprenant intègre toutes les caractéristiques qui font de lui ce qu'il est comme individu et ce qui le caractérise à son arrivée dans la formation.

\section{II.1.2. Le cours}

En ce qui concerne le cours, différents facteurs doivent être considérés afin de favoriser la motivation des apprenants en formation en ligne, comme sa flexibilité, sa pertinence, la qualité de son contenu, sa structure, son dynamisme, ses activités d'apprentissage et les évaluations qu'il propose. D'autres facteurs sont également à considérer, mais appartiennent plutôt à l'apprenant, au regard de cette dimension. Ainsi, la motivation de l'apprenant sera influencée par ses habitudes d'études, sa gestion du temps et du stress, son rapport au savoir, ses stratégies d'apprentissage, ses attentes, intentions et attitudes et la perception de sa compétence. Finalement, des auteurs parlent également de l'interaction de l'apprenant avec le contenu du cours, de sa participation et de sa présence cognitive. Nous regroupons ses aspects sous le facteur «niveau d'engagement ${ }^{4}$ ".

\section{II.1.3. Le formateur}

À l'étude des diverses recherches, certains facteurs relatifs au formateur ressortent à l'égard de la motivation de l'apprenant en ligne. Nous avons regroupé les différents résultats obtenus en quatre facteurs: les pratiques pédagogiques du formateur, ses compétences professionnelle, pédagogique et technologique, le soutien qu'il offre aux étudiants et sa participation dans le cours. La motivation de l'apprenant est également influencée par certains facteurs qui lui appartiennent au regard de cette dimension. Il s'agit de la qualité et des délais des interactions, ainsi que des attentes, intentions et attitudes que l'apprenant aura au regard du formateur.

\section{II.1.4. La technologie}

La motivation de l'apprenant est également influencée par la perception de certains facteurs relatifs à la technologie. En effet, la qualité de la technologie, son utilité (pertinence ou valeur), sa facilité d'utilisation et de navigation, son infrastructure technique et ses caractéristiques, ainsi que la diversité des outils qu'elle offre constituent des facteurs ayant un impact sur la motivation de l'apprenant. Du côté de l'apprenant, la perception de sa compétence jouera aussi sur sa motivation à apprendre en ligne, ainsi que ses attentes, intentions et attitudes au regard de la technologie.

\section{II.1.5. Autres apprenants}

Finalement, la perception de l'apprenant des attitudes de ses collègues agira sur sa motivation. Nous croyons également que sa perception de leur participation serait également un facteur pouvant influencer sa motivation, même si aucun auteur ne soulève 
ce dernier. En ce qui concerne les facteurs relatifs à l'apprenant, son intégration sociale, ses interactions avec les autres apprenants et la collaboration qu'il vivra avec ces derniers peuvent influencer la motivation de l'apprenant en ligne. Finalement, comme pour les trois autres dimensions, les attentes, intentions et attitudes que l'apprenant manifestera à l'égard de ses collègues auront également une influence sur sa motivation.

\section{II.2. L'approche et l'outil de collecte sélectionnés : des choix méthodologiques pour accéder à l'expérience}

Pour accéder à l'expérience, nous puiserons à des données narratives, au discours des acteurs. En ce sens, nous nous inscrivons dans le courant de la recherche qualitative qui permet de découvrir, d'explorer et de décrire certains phénomènes afin d'en avoir une compréhension plus approfondie, « animé[e] du désir de comprendre le sens de la réalité des individus " (Savoie-Zajc, 2004, p. 126). L'approche compréhensive, qui avance que toute personne peut accéder au vécu et au ressenti d'une autre (Mucchielli, 2004), nous apparait centrale dans notre démarche. Caractérisée par la quête de sens, elle tient compte des perceptions, sensations et impressions du participant au regard de sa réalité et est attentive aux significations qu'il lui attribue (Ibid.).

En ce qui concerne l'outil de collecte, l'entrevue constitue celui qui est le plus utilisé dans les recherches qualitatives (Fortin, 2010). Moyen privilégié pour mieux comprendre l'autre, elle permet d'établir un contact direct entre le chercheur et son participant (Ibid.). Dans notre démarche exploratoire, l'entrevue constitue donc un choix judicieux. L'entrevue semi-dirigée en recherche qualitative prend généralement la forme d'une discussion assez informelle (Ibid.), mais la structure est plus contrôlée (Savoie-Zajc, 2004). Nous postulons que celle-ci est la plus appropriée pour nous permettre de saisir le sens de l'expérience de l'apprenant en formation en ligne.

\section{II.3. Une clientèle ciblée : celle de la formation à l'enseignement}

31 Pour les besoins de cette recherche exploratoire, nous avons spécifiquement choisi, comme clientèle, les étudiants en formation à l'enseignement. Au Québec, la formation en ligne apparait comme une solution possible pour offrir des voies de qualifications aux futurs enseignants, en région plus éloignée, tant en formation initiale à l'enseignement qu'en formation continue.

À l'Université de Sherbrooke, trois programmes en ligne permettent aux étudiants d'effectuer une formation de deuxième cycle à l'enseignement : 1) la maitrise régulière ou qualifiante $^{5}$ en enseignement au secondaire; 2) la maitrise en adaptation scolaire et sociale et; 3) la maitrise en enseignement et le diplôme de deuxième cycle en enseignement au préscolaire et au primaire ${ }^{6}$.

\section{La réalisation de la recherche exploratoire: l'analyse préliminaire d'une entrevue}

Pour cette préexpérimentation, nous avons réalisé une entrevue auprès de Josée, une étudiante à la maitrise régulière en enseignement au secondaire. Le canevas préparé pour cette entrevue comportait six questions générales, dont deux principales : il s'agissait 
principalement d'amener l'étudiante à partager son expérience dans le cadre de deux cours en ligne. Dans cette partie, nous effectuons l'analyse préliminaire de cette entrevue, puis nous terminons par un retour critique sur celle-ci.

L'analyse qui prévaut avec ce type de recherche consiste à saisir le sens des données recueillies (Savoie-Zajc, 2004). Il s'agit d'un processus d'organisation et d'interprétation de ces données, de façon à en dégager des thèmes et catégories pour décrire le phénomène (Fortin, 2010). L'analyse effectuée ici se fera à partir de deux méthodes. D'abord, nous utilisons celle de la logique inductive délibératoire (Savoie-Zajc, 2004), c'est-à-dire que nous catégorisons les réponses obtenues à partir du cadre-synthèse présenté plus tôt. D'ailleurs, lorsque d'autres facteurs émergent, nous bonifions ce cadre en conséquence (Ibid.). Si d'autres informations que les étudiants ressortent au regard de leur expérience n'entrent pas dans ce cadre, nous utilisons par la suite la méthode inductive modérée (Ibid.) qui permet de laisser émerger d'autres catégories.

\section{III.1. L'analyse préliminaire de l'expérience de Josée en formation en ligne}

L'entrevue réalisée avec Josée s'est déroulée en ligne. Pour effectuer l'analyse préliminaire de celle-ci, nous avons retranscrit le verbatim issu de l'enregistrement et utilisé un code de couleur pour identifier les dimensions du cours concernées par ses propos. À partir du cadre-synthèse, nous avons créé ici le schéma de sa propre expérience (figure 2) en indiquant les aspects qui ressortent de son entrevue. Nous reprenons ici brièvement chacune de ces dimensions : l'apprenant, le cours, le formateur, les autres apprenants et la technologie.

Figure 2 : Portrait des facteurs ayant un impact sur la motivation de Josée dans son expérience de formation en ligne

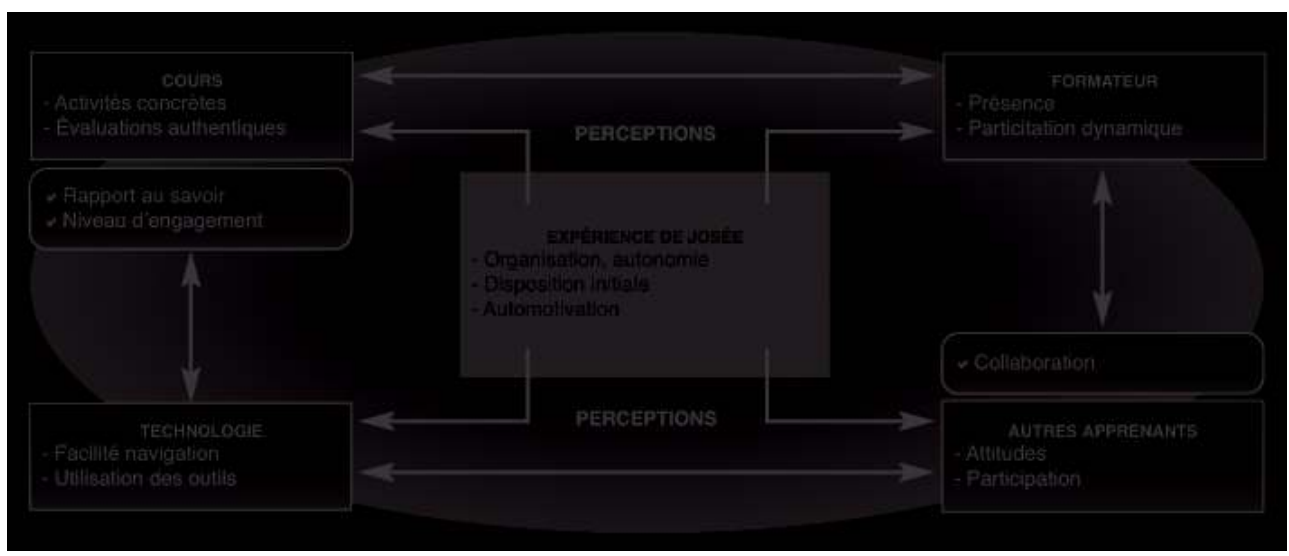

D'abord, la dimension centrale de ce schéma est ce qui caractérise Josée comme apprenante. Celle-ci se qualifie comme étant organisée et autonome et considère qu'il s'agit de conditions facilitantes dans ce type de formation. Également, certaines caractéristiques inhérentes à la formation en ligne, comme le fait de pouvoir étudier de la maison et de choisir elle-même ses heures d'étude, ont constitué des incitatifs importants à son choix de programme. Cette information nous amène à penser qu'elle possédait de bonnes dispositions initiales dès le départ. Elle considère d'ailleurs que c'est dans sa nature d'être motivée et de ne pas abandonner: «je me convaincs tout le temps que je 
dois essayer, que je dois le faire ". Ces propos nous amènent à percevoir chez elle une forte capacité à l'automotivation qui est également un facteur identifié dans notre cadresynthèse.

En ce qui concerne la dimension du cours, Josée mentionne que ce n'est pas vraiment le contenu qui lui fait apprécier ou non une formation, mais plutôt le formateur. Toutefois, elle relève certains facteurs qui ont un impact sur sa motivation, tels que des éléments de contenu trop théoriques et peu d'applications concrètes pour la soutenir dans sa profession d'enseignante : « je suis rendue sur le terrain tous les jours, je voulais des trucs pour m'aider, pas de la théorie ». Elle dit également qu'elle apprécie les travaux concrets et appliqués, qui lui permettent de réinvestir ses nouveaux apprentissages: «cela me permet de savoir si j'ai vraiment compris ce que j'ai appris. Ce n'est pas juste recracher les informations ». Pour Josée, au regard de notre cadre-synthèse, nous parlons donc d'activités concrètes et d'évaluations authentiques. L'étudiante parle aussi de son niveau d'engagement lorsqu'elle n'est pas motivée, en soulevant que dans ces cas, elle n'est pas aussi ouverte aux apprentissages : «C'est comme si c'était un gros poids tout à coup. Mais je fais quand même les travaux et tout là, mais on dirait que ça ne me tente pas. Donc, je fais le minimum ». Ces propos mettent l'accent sur les conséquences que peut avoir l'absence de motivation sur l'engagement cognitif et ainsi, sur l'apprentissage. Elle mentionne quand même que pour elle, les apprentissages sont prioritaires, mais c'est la note qui fait la différence à la fin. Ces éléments d'informations, qui nous paraissent importants, correspondent, selon notre cadre, aux facteurs relatifs à l'apprenant à l'égard de la dimension du cours (rapport au savoir, niveau d'engagement).

L'analyse de l'entrevue avec Josée révèle toute l'importance que cette étudiante accorde à la dimension du formateur en mentionnant que dans une formation en ligne, c'est sa présence, son dynamisme et ses réponses rapides, enjouées et humoristiques qui lui font apprécier le cours et qui lui donne le gout d'apprendre : « on dirait qu'avec ça, je carbure, [alors] je suis motivée et j'essaie tout le temps. [...] Je pense que c'est vraiment ce qui fait la différence ». Ces réponses sont directement associées aux facteurs: présence et participation du formateur dans notre cadre-synthèse.

L'étudiante parle peu de la dimension des autres apprenants : elle se définit elle-même comme une personne qui ne discute pas beaucoup en classe et la solitude ne la dérange pas. Elle dit toutefois apprécier les forums dédiés à l'entraide : « ça rend les choses un peu plus vraies".

40 En ce qui concerne la technologie, cette étudiante relève que l'organisation des cours par section sur la plateforme est facilitante (renvoie à la facilité de navigation) et critique l'utilisation qui est faite des forums de discussion dans certains cours. En effet, elle déplore le fait que des formateurs utilisent cet outil à d'autres fins que la discussion ou les échanges. Dans notre cadre-synthèse, la dimension de la technologie parle du facteur de la «diversité des outils» et de "l'utilité » de la technologie en général, mais non de la pertinence de l'utilisation des outils ciblés. Cette entrevue nous permet de constater l'importance que ce facteur peut avoir et ce dernier pourra être ajouté au cadre-synthèse.

41 Ce qui ressort principalement de cette analyse préliminaire, c'est le fait que la dimension relative à l'apprenant, centrale dans notre modèle, est la base par laquelle l'étudiante définit son l'expérience et à partir de laquelle celle-ci perçoit les autres dimensions. En effet, Josée semble apprécier sa formation et vivre son expérience selon la lunette de ce qu'elle est comme personne, de ce qui la caractérise. Par exemple, c'est probablement parce qu'elle est organisée et qu'elle possède de bonnes dispositions au départ qu'elle 
s'investit et apprécie la formule autonome et c'est aussi peut-être parce qu'elle est plus solitaire que le peu d'interactions avec ses collègues ou le formateur ne semble pas lui manquer. Ainsi, le schéma nous permet d'avoir une vue d'ensemble des facteurs ayant un impact sur la motivation de Josée dans son expérience en formation en ligne.

\section{III.2. Un retour critique sur l'entrevue exploratoire réalisée}

Nous portons maintenant un regard critique sur notre démarche comme intervieweuse puisque l'attitude du chercheur est déterminante de la qualité des données qui émergent. Dans notre cas, l'entrevue réalisée avec cette étudiante s'est déroulée dans un climat de respect et d'écoute. Nous constatons toutefois que la formulation des questions et des relances n'était pas toujours neutre. Également, par l'analyse préliminaire de l'entrevue de Josée, nous constatons que certains aspects auraient dû être approfondis. En effet, à quelques moments lors de l'entrevue, nous aurions dû poser davantage de questions afin de clarifier ce que l'étudiante insinuait. Lors d'une prochaine entrevue, nous serons plus prudente pour la formulation des questions et relances et serons attentive aux propos soulevés qui mériteraient d'être approfondis.

\section{Les suites méthodologiques à envisager pour la démarche doctorale}

La démarche exploratoire présentée ici nous permet de relever certains choix méthodologiques à envisager pour notre thèse. À cette étape-ci de nos recherches, nous confirmons la pertinence de notre question générale et envisageons de créer notre échantillon sur la base d'étudiants inscrits à l'intérieur d'un seul programme. L'outil de collecte demeurerait l'entrevue, mais nous songeons à lui donner une forme plus libre. Une entrevue non directive pourrait donc être réalisée auprès d'un petit nombre d'étudiants et ces derniers pourraient nous parler librement de leur expérience de formation en ligne. Tout en étant à l'écoute de leurs propos, nous poserions, tout au long de l'entrevue, de multiples questions de relance afin de les amener à déplier les implicites, au sens de faire sortir les plis, pour mieux saisir la subjectivité (Gate, 2004). Il pourrait d'ailleurs s'avérer pertinent de suivre ces derniers pendant toute la durée de la session afin de voir comment leur expérience évolue dans le temps. Ces entrevues réalisées à différents moments permettraient, du même coup, une double sollicitation, c'est-à-dire de retourner auprès de l'étudiante " pour faire livrer le sens que [celle-ci] pourrait avoir laissé dans l'ombre » (Dumoulin, 2010, p. 105).

\section{En conclusion : les retombées anticipées}

Alors que la problématique du taux élevé d'abandons en enseignement supérieur en formation en ligne apparait importante à considérer, l'idée d'accéder à l'expérience de l'apprenant et à la façon dont se manifeste sa motivation à l'intérieur de celle-ci nous apparait pertinente comme piste de recherche à explorer. C'est donc sur la base de notre cadre-synthèse que l'analyse préliminaire d'une entrevue réalisée auprès d'une étudiante en formation en ligne à l'enseignement a été présentée ici. Cette recherche exploratoire nous a permis de dresser le portrait de la façon dont se manifeste sa motivation dans son 
expérience de formation en ligne et nous a permis de constater que la dimension de l'apprenant est à la base de ses perceptions, ce qui lui permet de donner une signification à l'ensemble de son expérience.

Nous supposons donc que notre recherche pourra avoir de multiples retombées positives. En effet, en cherchant à mieux comprendre l'expérience motivationnelle des apprenants universitaires en formation en ligne, de nouvelles possibilités d'intervention et d'adaptations de l'enseignement pourront émerger. Cela nous permettra d'enrichir et de valider le contenu du cadre-synthèse présenté ici. Sur la base de ce dernier, nous pourrons alors bâtir un cadre susceptible d'intervenir favorablement sur la motivation des apprenants universitaires en formation en ligne. Nous supposons que ce dernier constituerait un outil pour sensibiliser les formateurs en ligne à l'importance de considérer la motivation des apprenants pour une plus grande persévérance et une réussite accrue.

\section{BIBLIOGRAPHIE}

Allen, I. E. et Seaman, J. (2008). Staying the course: Online education in the United States. Needham: Sloan Consortium.

Aragon, S. R. et Johnson, E. S. (2008). Factors influencing completion et noncompletion of community college online courses. American Journal of Distance Education, 22(3), 146-158.

Bates, T. (2011). 2011 outlook for online learning et distance education. Ontario: Ontario's Distance Education et Training Network.

Bekele, T. A. (2010). Motivation et Satisfaction in Internet-Supported Learning Environments: A Review. Educational Technology et Society, 13(2), 116-127.

Castles, J. (2004). Persistence et the adult learner: factors affecting persistence in Open University students. Active learning in higher education, 5(2), 166-179.

Chen, K.-C. et Jang, S.-J. (2010). Motivation in online learning: testing a model of selfdetermination theory. Computers in Human Behavior, 26(4), 741-752.

Dumoulin, M.-J. (2010). La restructuration de l'expérience chez trois enseignantes débutantes en contexte d'accompagnement mentoral. Thèse de doctorat en éducation, Université de Sherbrooke, Sherbrooke.

Fortin, M.-F. (2010). Fondements et étapes du processus de recherche : méthodes quantitatives et qualitatives (2e éd.). Montréal : Chenelière éducation (1re éd. 2006).

Gate, J.-P. (2004). À la rencontre des pratiques d'apprentissage : réflexions à propos d'un modèle d'entretien de recherche à visée métacognitive. Recherches et éducation, 8.

Grayson, P. (2003). Les recherches sur le maintien et la diminution des effectifs étudiants. Fondation canadienne des bourses d'études du millénaire : Montréal. 
Ivankova, N. V. et Stick, S. L. (2007). Students' persistence in a distributed doctoral program in educational leadership in higher education: A mixed methods study. Research in Higher Education, 48(1), 93-135.

Keller, J. M. (1983). Motivational design of instruction. In C. M. Reigeluth (dir.), Instructional theories and models: an overview of their current status (p. 386-434).New York: Lawrence Erlbaum Associates.

Kember, D. (1989). A longitudinal-process model of drop-out from distance education. Journal of Higher Education, 60(3), 278-301.

Kim, S. (2008). Étude des représentations du personnel enseignant à l'égard de ses pratiques d'ordre technologique et pédagogique actuelles et de celles qui pourraient favoriser la mise en cuvre d'un dispositif de formation à distance à l'Institut de Technologie du Cambodge. Thèse de doctorat en éducation, Université de Sherbrooke, Sherbrooke.

Kim, S.-M., Barbier, R. et Verrier, C. (2007). Implication et enseignement en ligne. Communication présentée au Congrès international AREF (Actualité de la Recherche en Éducation et en Formation), Strasbourg.

Lakhal, S. et Khechine, H. (2014). Les déterminants de la persévérance des étudiants adultes dans les cours en ligne. Communication présentée au Colloque Cirta, Sherbrooke, 15-16 octobre 2014.

Lee, Y. et Choi, J. (2011). A review of online course dropout research: implications for practice and future research. Educational Technology Research and Development, 59(5), 593-618.

Levy, Y. (2007). Comparing dropouts and persistence in e-learning courses. Computers and Education, 48(2), 185-204.

Lim, D. H. (2004). Cross cultural differences in online learning motivation. Educational Media International, 41(2), 163-173.

Metzger, J.-L. et Delalonde, C. (2005). Innovation pédagogique et collectif dans la formation en ligne en France et aux États-Unis. Communication et langages, 144, 25-36.

Mucchielli, A. (2004). Dictionnaire des méthodes qualitatives en sciences humaines (2e éd.). Paris : Arman Colin (1re éd. 1996).

Mullen, G. E. and Tallent-Runnels, M. K. (2006). Student outcomes et perceptions of instructors'demands and support in online and traditional classrooms. Internet and Higher Education, 9(4), 257-266.

Park, J. (2007). Factors related to learner dropout in online learning. In F. M. Nafukho, T. H. Chermack and C. M. Graham (Dir.), Proceedings of the 2007 Academy of Human Resource Development Annual Conference (p. 25-1-25-8). Indianapolis, IN: Ahrd.

Park, J. H. and Choi, H. J. (2009). Factors Influencing Adult Learners' Decision to Drop Out or Persist in Online Learning. Educational Technology and Society, 12(4), 207-217.

Perriault, J. (2002). L'accès au savoir en ligne. Paris : Odile Jacob.

Pintrich, P. R. (1990). Implications of psychological research on student learning and college teaching for teacher education. In W. R. Houston (dir.), Handbook of research on teacher education (p. 826-857). New York : MacMillan Publishing.

Poellhuber, B. et Chomiene, M. (2006). L'amélioration de la persévérance dans les cours de formation à distance : les effets de l'encadrement et de la collaboration. Rosemont : Cégep à distance. 
Prata, A. et Lopes, P.-F. (2005). Online multimedia educational application for teaching multimedia contents: An experiment with students in higher education. In P. Darbyshire (dir.), Instructional Technologies: Cognitive Aspects of Online Programs (p. 31-72). Hershey, PA : IRM Press.

Racette, N. (2009). La conception d'un programme motivationnel destiné aux cycles supérieurs en formation à distance. Revue de l'éducation à distance, 23(2), 25-50.

Rovai, A. P. (2003). In search of higher persistence rates in distance education online programs. The Internet and Higher Education, 6(1), 1-16.

Ruel, G. (2010). Persistance scolaire en formation à distance. DistanceS, 12(1), 27-43.

Sauve, L., Debeurme, G., Martel, V., Wright, A., Hanca, G., Fournier, J. et Castonguay, M. (2007). L'abandon et la persévérance aux études postsecondaires. Québec : SAMI-persévérance.

Savoie-Zajc, L. (2004). La recherche qualitative/interprétative en éducation. In T. Karsenti et L. Savoie-Zajc (dir.), La recherche en éducation : étapes et approches (p. 123-150). Sherbrooke: Éditions du CRP.

Selvi, K. (2010). Motivating factors in online courses. Procedia Social and Behavioral Sciences, 2(2), 819-824.

Thouraya, D. (2007). Guider les étudiants universitaires vers l'autorégulation dans leur apprentissage en ligne. Revue internationale des technologies en pédagogie universitaire, 4(1), 20-31.

Tinto, V. (1975). Dropout from higher education: A theoretical synthesis of recent research. Review of Educational Research, 45(1), 89-125.

Viau, R. (2009). La motivation à apprendre en milieu scolaire. Québec : Éditions du Renouveau Pédagogique Inc.

Yoo, S. J. et Huang, W. D. (2013). Engaging Online Adult Learners in Higher Education: Motivational Factors Impacted by Gender, Age, and Prior Experiences. The Journal of Continuing Higher Education, 61, 151-164.

Weiner, B. (1992). Human motivation. Newbury Park: Sage.

\section{NOTES}

1. Traduction personnelle.

2. Keller (1983) ; Pintrich (1990); Viau (2009); Weiner (1992).

3. La liste de ces études est accessible à l'adresse suivante: http://slideshare.com/ carolinebourque

4. Il est à noter que selon Salomon (1983, dans VIAU, 2009), «l'engagement cognitif correspond au degré d'effort mental que l'élève déploie lors de l'exécution d'une activité pédagogique » (p. 52).

5. La maitrise régulière est une formation continue pour les étudiants possédant déjà un brevet d'enseignement (équivaut à une licence en France). La maitrise qualifiante est une formation initiale pour ceux possédant un baccalauréat dans une discipline spécifique.

6. Les étudiants qui y sont inscrits sont issus du baccalauréat en enseignement au préscolaire et au primaire et, pour la majorité, sont déjà en fonction dans une école.

7. Rappelons que notre question générale se formule ainsi: «Comment la motivation de l'apprenant universitaire se manifeste-t-elle dans son expérience de formation en ligne?». 


\section{RÉSUMÉS}

Alors qu'un taux élevé d'abandon est observé en formation en ligne, une recension a permis de cibler la motivation comme variable importante. Cet article présente une entrevue exploratoire réalisée dans le cadre d'une recherche doctorale. L'analyse préliminaire menée sur la base d'un cadre-synthèse de motivation en formation en ligne permet de dresser un portrait de l'expérience d'une apprenante en formation à l'enseignement et de la façon dont sa motivation se manifeste à l'intérieur de celle-ci.

A high dropout rate is observed in online training, and analysis has targeted motivation as important variable. This article presents an exploratory interview as part of a doctoral study. The preliminary analysis conducted on the basis of a framework synthesis of motivation in online training (or learning) helps paint a picture of the experience of a learner in teacher education and how motivation is manifested within this person.

\section{INDEX}

Mots-clés : expérience, formation à l'enseignement, formation en ligne, motivation Keywords : experience, motivation, online learning, teacher education.

\section{AUTEURS}

\section{CAROLINE BOURQUE}

Doctorante en éducation, Faculté d'éducation, Université de Sherbrooke

\section{CÉLINE GARANT}

Ph.D., Professeure, Faculté d'éducation, Université de Sherbrooke 